\title{
Modulation of corticospinal excitability by transcranial magnetic stimulation in children and adolescents with autism spectrum disorder
}

\author{
Lindsay M. Oberman 1,2,3,4 *, Alvaro Pascual-Leone ${ }^{1}$ and Alexander Rotenberg ${ }^{1,2}$ * \\ ${ }^{1}$ Department of Neurology, Berenson-Allen Center for Noninvasive Brain Stimulation, Beth Israel Deaconess Medical Center- Harvard Medical School, Boston, \\ MA, USA \\ ${ }^{2}$ Neuromodulation Program and Division of Epilepsy and Clinical Neurophysiology, Department of Neurology, Boston Children's Hospital - Harvard Medical \\ School, Boston, MA, USA \\ ${ }^{3}$ Neuroplasticity and Autism Spectrum Disorder Program, E. P. Bradley Hospital, East Providence, RI, USA \\ ${ }^{4}$ Department of Psychiatry and Human Behavior, Warren Alpert Medical School of Brown University, East Providence, RI, USA
}

\section{Edited by:}

Peter G. Enticott, Deakin University, Australia

\section{Reviewed by:}

Paul Croarkin, Mayo Clinic, USA Melissa Kirkovski, Monash University, Australia

\section{*Correspondence:}

Lindsay M. Oberman, Neuroplasticity and Autism Spectrum Disorder Program, E. P. Bradley Hospital, 1011 Veterans Memorial Parkway, East Providence, RI 02915, USA e-mail: loberman@lifespan.org; Alexander Rotenberg, Neuromodulation Program and Division of Epilepsy and Clinical Neurophysiology, Department of Neurology, Boston Children's Hospital - Harvard Medical School, 300 Longwood Avenue, Boston, MA 02115, USA

e-mail: alexander.rotenberg@ childrens. harvard.edu
The developmental pathophysiology of autism spectrum disorders (ASD) is currently not fully understood. However, multiple lines of evidence suggest that the behavioral phenotype may result from dysfunctional inhibitory control over excitatory synaptic plasticity. Consistent with this claim, previous studies indicate that adults with Asperger's Syndrome show an abnormally extended modulation of corticospinal excitability following a train of repetitive transcranial magnetic stimulation (rTMS). As ASD is a developmental disorder, the current study aimed to explore the effect of development on the duration of modulation of corticospinal excitability in children and adolescents with ASD. Additionally, as the application of rTMS to the understanding and treatment of pediatric neurological and psychiatric disorders is an emerging field, this study further sought to provide evidence for the safety and tolerability of rTMS in children and adolescents with ASD. Corticospinal excitability was measured by applying single pulses of TMS to the primary motor cortex both before and following a $40 \mathrm{~s}$ train of continuous theta burst stimulation. 19 highfunctioning males ages 9-18 with ASD participated in this study. Results from this study reveal a positive linear relationship between age and duration of modulation of rTMS aftereffects. Specifically we found that the older participants had a longer lasting response. Furthermore, though the specific protocol employed typically suppresses corticospinal excitability in adults, more than one third of our sample had a paradoxical facilitatory response to the stimulation. Results support the safety and tolerability of rTMS in pediatric clinical populations. Data also support published theories implicating aberrant plasticity and GABAergic dysfunction in this population.

Keywords: autism spectrum disorders, transcranial magnetic stimulation, development, plasticity, GABA, theta burst stimulation

\section{INTRODUCTION}

Autism spectrum disorder (ASD) is diagnosed clinically, based on the key symptoms including qualitative impairments in social communication and the presence of restricted and repetitive behaviors (APA, 2013). However, the variability of the clinical phenotype of ASD is quite large and symptoms can manifest over a range of ages in childhood. Thus, ASD diagnosis can be challenging and is often not made until 3-5 years of age. For this reason, a physiologic ASD biomarker is highly desirable.

Several lines of evidence suggest that an impairment of GABAergic transmission may be critical in the pathophysiology of ASD (see Coghlan et al., 2012 for a review). GABA plays a key role in regulating neuronal excitability via feedback and feed-forward inhibition (Sutor and Luhmann, 1995; Petroff, 2002; Madsen et al., 2008; Huang, 2009). While in the mature brain GABA acts as an inhibitory neurotransmitter, during the embryonic and the perinatal period, GABA is excitatory (Cherubini et al., 1991). It is hypothesized that at least some forms of autism result from an imbalance between excitation and inhibition in local circuits involved in sensory, mnemonic, social, and emotional processes (Rubenstein and Merzenich, 2003; Markram and Markram, 2010). Ben-Ari et al. (2012), for instance, suggest that a dysfunction in the shift of GABA from excitation to inhibition may contribute to this imbalance.

Empirical support for the role of aberrant GABA signaling in the pathophysiology of ASD comes from both human and animal model research. A recent study conducted by Tyzio et al. (2014) found that GABA had excitatory action in two animal models of ASD [rats exposed to valproate in utero (VPA) and mice carrying the fragile X mutation (FRX)]. Furthermore, maternal pretreatment with bumetanide, forcing the shift of GABA in the offspring from excitatory to inhibitory, resulted in the restoration of typical electrophysiological and behavioral phenotypes in affected animals (Tyzio et al., 2014). 
Human studies have found reduced GABA receptor expression (Fatemi et al., 2009a,b, 2010) as well as a 50\% reduction in enzymes that synthesize GABA [glutamic acid decarboxylase (GAD) 65 and 67; Fatemi et al., 2002; Yip et al., 2007] in individuals with ASD. Furthermore, a recent study (Gaetz et al., 2014) identified significant reduction in the GABA MRS signal in the motor cortex of patients with ASD, and a marginally significant $(p=0.054)$ positive correlation between the GABA signal and age. Among the many roles of GABAergic circuits during development, one is lateral inhibition across neighboring minicolumns in the cortex. Consistent with an impairment in GABAergic transmission, postmortem studies have found a reduction in the horizontal spacing between minicolumns (Casanova et al., 2002). These abnormalities in the GABA system may directly contribute to altered anatomical and functional connectivity, and suggest a mechanism underlying the neurological and behavioral phenotype of ASD (Blatt, 2011).

In addition to and perhaps as a consequence of excitation/inhibition imbalance, recent studies in both human and animal models implicate synaptic plasticity mechanisms in the pathophysiology of ASD (see Oberman et al., in press). While most synaptic plasticity data in ASD are derived from in vitro rodent brain slice models, direct measures of circuit level plasticity in humans can be obtained by transcranial magnetic stimulation (TMS) paradigms (Ziemann, 2004; Huang et al., 2005; Thickbroom, 2007; Huerta and Volpe, 2009). In TMS, the cortex is stimulated focally by small intracranial electrical currents that are generated by a powerful and fluctuating extracranial magnetic field (Barker et al., 1985; Kobayashi and Pascual-Leone, 2003; Hallett, 2007). A number of experimental TMS measures of brain plasticity have been introduced, and provide the only noninvasive capacity to measure human phenomena that closely resemble long-term potentiation (LTP) and long-term depression (LTD). TMS is safe and well-tolerated, even in pediatric populations, if appropriately guidelines and recommendations are followed (Garvey and Gilbert, 2004; Rajapakse and Kirton, 2013).

Single-pulse TMS combined with EMG, EEG, fMRI, or other brain imaging methods can be used to quantify cortical reactivity before and following a given intervention (Pascual-Leone et al., 2011) providing an index of brain plasticity in response to said intervention. Recently, patterned bursting protocols have been developed that mimic paradigms used to assess synaptic plasticity in animal models (Huang et al., 2005, 2008). Specifically, theta burst stimulation (TBS) involves application of three bursts of $50-\mathrm{Hz}$ rTMS repeated every $200 \mathrm{~ms}$ either continuously for a total of $40 \mathrm{~s}$ or intermittently (every $8 \mathrm{~s}$ ) for about $3 \mathrm{~min}$. When applied to the motor cortex, continuous TBS (cTBS) and intermittent TBS (iTBS) result in depression and potentiation of cortical reactivity as indexed through suppression and facilitation of motor evoked potentials (MEPs), respectively (Huang et al., 2005). Results from animal and human studies indicate that TBS modulatory effects on cortical reactivity reflect synaptic plasticity mechanisms (Cardenas-Morales et al., 2011). Specifically, and relevant to the present experiment, published data suggest that cTBS leads to enhancement of GABAergic inhibition (Stagg et al., 2009; Benali et al., 2011).

Notably, compared to other rTMS protocols, TBS has the advantage of lower stimulation intensities and shorter durations than conventional protocols making this protocol more suitable for use in clinical and pediatric populations. The safety and tolerability of this protocol has recently been evaluated and shown to be safe in healthy children and in children with Tourette Syndrome (Wu et al., 2012).

In a recent study (Oberman et al., 2012) we used the cTBS paradigm in 20 adults with Asperger's syndrome (high functioning ASD), and found them to show greater and longer-lasting suppression of cortical reactivity in the motor cortex following cTBS as compared to age-, gender-, and IQ-matched controls. The latency to return to baseline following TBS was on average between 80 and $90 \mathrm{~min}$ in the ASD group compared to 25-30 min in the controls. This finding was confirmed in a separate cohort of 15 individuals (Oberman et al., 2012). Interestingly, and consistent with other studies, there was no significant group difference in measures of basic excitability as assessed by resting and active motor threshold (Theoret et al., 2005; Oberman et al., 2012; Enticott et al., 2013) or response to single pulse TMS (Oberman et al., 2012). Thus, the excessive modulation of excitability in response to stimulation (a putative measure of plasticity) is not primarily attributable to differences in baseline excitability.

In the current study, we extended our age range to include data from 19 children and adolescents with high-functioning ASD (HF ASD) to explore the effect of development on the response to the cTBS paradigm. As the application of rTMS to the understanding and treatment of pediatric neurological and psychiatric disorders is an emerging field (Frye et al., 2008; Croarkin et al., 2011), this study additionally aimed to provide evidence for the safety and tolerability of TBS in HF children and adolescents with ASD.

\section{MATERIALS AND METHODS PARTICIPANTS}

We studied 19 males with HF ASD, age 9-18 years (See Table 1 for demographic characteristics of the sample). All participants gave informed consent or assent, which was also obtained from a parent or guardian to participate in the study. The study was reviewed and approved by the institutional review board at Boston Children's Hospital. Participants were recruited through local community advertisement. All participants had IQ $>80$ based on the Weschler Abbreviated Scale of Intelligence (WASI). All met DSM-IV-TR criteria for Autism, Asperger's Syndrome or PDD-NOS, and met criteria for ASD on the Autism Diagnostic Observation Schedule, Module 4 (ADOS). Some participants also had comorbid symptoms including inattention, anxiety, irritability, and obsessive-compulsive behaviors (See Table 1). All participants were given a comprehensive neurological exam by a board-certified pediatric neurologist (Alexander Rotenberg) to confirm normal gross motor and fine motor function. Lastly, all participants were screened following published recommendations (Rossi et al., 2009) to ensure that they did not have any condition that would put them at greater risk of an adverse event related to TMS (e.g., a personal or immediate family history of epilepsy).

\section{STIMULATION AND RECORDING}

To evaluate modulation of corticospinal excitability (a putative index of cortical plasticity mechanisms) and specifically GABAergic inhibition, cTBS was applied to the primary motor cortex. The 
Table 1 | Sample characteristics.

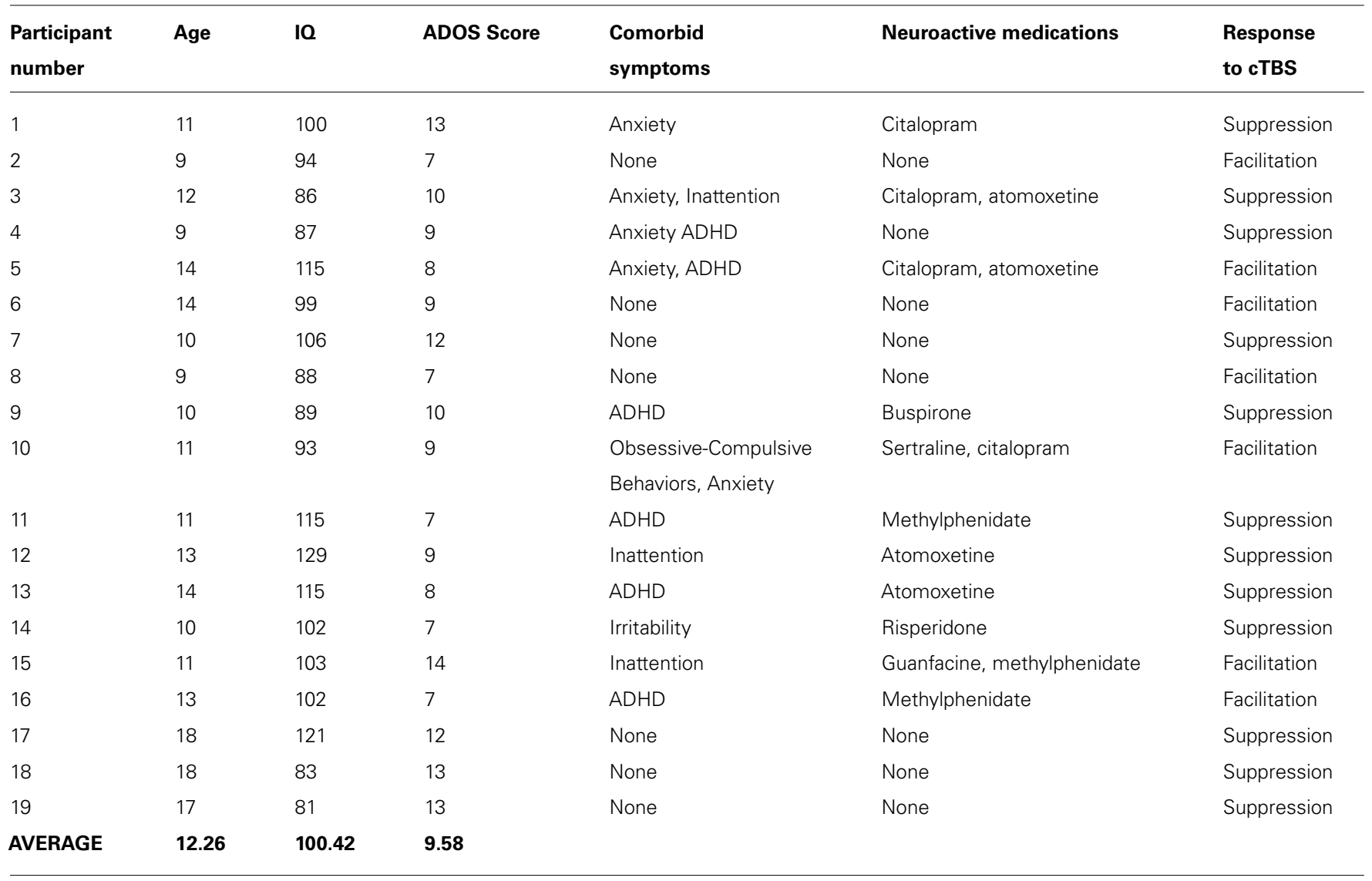

cTBS paradigm used in the current study was identical to that described by Huang et al. (2005) and applied in previous studies in our laboratory (Oberman et al., 2010, 2012). The protocol consisted of three pulses of $50 \mathrm{~Hz}$ stimulation repeated at 200$\mathrm{ms}$ intervals for $40 \mathrm{~s}$ (for a total of 600 pulses) at an intensity of $80 \%$ of active motor threshold (AMT). Corticospinal excitability was assessed prior to and following cTBS by measuring peak-topeak amplitude of MEPs induced in the contralateral first dorsal interosseus (FDI) muscle in response to single-pulse TMS. These single pulses were applied at a rate of approximately $0.1 \mathrm{~Hz}$ (a random jitter of $\pm 1 \mathrm{~s}$ was introduced to avoid any train effects). Three batches of 10 MEPs were recorded prior to cTBS and used as a baseline. Beginning at 5 min following cTBS, batches of 10 MEPs were measured at periodic intervals (5, 10, 15, 20, 30, 40, $50,60,70,80,90,105$, and $120 \mathrm{~min}$ ) until the MEPs returned to baseline levels to track changes in MEP amplitude over time. The participant was asked to remain relaxed during the entire study. Muscle activity was monitored throughout the session with EMG surface electrodes. TMS was only applied when the EMG signal indicated that the participant's FDI muscle was in a relaxed state. Any trials where the participant voluntarily contracted the muscle within $1000 \mathrm{~ms}$ of the TMS pulse were not included in the analysis.

To measure TMS induced MEPs, EMG surface electrodes were placed in a belly tendon montage over the FDI muscle of participants' right hands. Raw signals were amplified and bandpass-filtered between 20 and $2000 \mathrm{~Hz}$. EMG signals were sampled at a rate of $5000 \mathrm{~Hz}$. All stimulation (single-pulse TMS and TBS) was delivered using a hand-held $70 \mathrm{~mm}$ figure-ofeight coil attached to a Magstim Super Rapid stimulator (The MagStim Company Ltd., Whitland, UK). The coil was placed tangentially to the scalp with the handle pointing posteriorly. All stimulation was applied over the hand area of the left motor cortex and individually localized for each participant based on the optimal position for eliciting MEPs in the right FDI. The stimulation intensity for baseline and post-TBS single pulses was set at $120 \%$ of each individual's resting motor threshold (RMT) while the TBS itself was delivered at $80 \%$ of AMT. RMT and AMT were defined following recommendation from the International Federation of Clinical Neurophysiology. RMT was defined as the minimum single-pulse TMS intensity required to induce an MEP in the contralateral FDI of $>50 \mu \mathrm{V}$ peak-to-peak amplitude on more than five out of ten consecutive trials while the target muscle was at rest. AMT was defined as the minimum single-pulse TMS intensity required to induce an MEP in the contralateral FDI of $>200 \mu \mathrm{V}$ peak-to-peak amplitude on more than five out of ten consecutive trials while the target muscle was held at approximately $20 \%$ of the maximal contraction. To precisely target the stimulation site (primary motor cortex) and keep the brain target constant throughout the stimulation session, we used a frameless 
stereotactic neuronavigation system (Brainsight, Rogue Research Inc., Montreal, QC, Canada).

\section{DATA ANALYSIS}

Data were analyzed using MatLab version 8.1 and SPSS version 22. Data analysis followed the methods described and applied in previous studies in our laboratory (Oberman et al., 2010, 2012). Average MEP amplitude values were calculated at baseline prior to TBS and starting five minutes after TBS and continuing until the average amplitude returned to within the 95\% confidence interval of the baseline amplitude and did not return to outside that interval on subsequent time-point measures. MEP amplitudes were standardized, forming a ratio of MEP amplitudes following TBS relative to average baseline MEP amplitude for each individual.

Cubic spline interpolation was used to create smooth curves through the data points. Spline interpolation is a piecewise continuous function defined by third-degree polynomials in the intervals of a limited range of known data points (in this case, the time-points at which MEP data were collected with batches of 10 single TMS pulses). The use of spline interpolation on TMS data has been validated (Borghetti et al., 2008) and used in previous studies to evaluate degree and duration of modulation of MEP amplitudes following cTBS (Freitas et al., 2011). As an index of the duration of the TBS-induced modulation of cortico-spinal excitability, we defined, for each participant, the time-point ("time to baseline") at which post-cTBS MEP amplitude returned to the average MEP amplitude at baseline, i.e., the time-point at which the spline crossed the MEP threshold.

A natural log transformation was applied to the data prior to analysis as tests of normality indicated that the data was significantly different than normal. A Pearson product-moment correlation coefficient was calculated to assess the degree of relationship between age and duration of response to cTBS.

\section{SIDE EFFECT MONITORING}

Immediately following the TMS session a side effects questionnaire was completed by the experimenter. Participants were asked to report whether they experienced any of the following side effects: headache, neck pain, scalp pain or irritation, difficulty hearing, thinking or concentrating, change in mood, or any other change or side effect they experienced. The experimenter also noted whether the participant experienced a syncopal event or seizure. The participant also received a call the day after the TMS session and was once again asked to report whether they experienced any of the above side effects or to report any other side effect they experienced after they left the hospital. If the participant reported any side effect either immediately following the stimulation or the following day, its severity and duration were documented.

\section{RESULTS}

\section{TMS SAFETY AND TOLERABILITY}

All participants tolerated TBS and single-pulse stimulation without serious adverse event. One participant had a mild headache after stimulation that was alleviated with a single acetaminophen dose. Two participants had mild fatigue after the session that resolved the following day. No other adverse events were reported.

\section{AGE-DEPENDENT RESPONSE TO cTBS}

A Pearson product-moment correlation coefficient was calculated to test the hypothesis that a linear relationship existed between age and duration of response to cTBS. As described above, response to the cTBS protocol was defined as duration of effect as defined by the number of minutes following cTBS before the participant returned to baseline excitability levels ("time-to-baseline"; $M=46.3 \mathrm{~min}, \mathrm{SD}=29.3 \mathrm{~min}$ ). The results of the analysis indicated that there was a significant positive linear relationship between age and duration of response $[r(17)=0.660, p<0.01$; Figure 1].

In addition to the planned analyses, it was noted that unlike our previous study that included only adult participants, a third of the participants ( 7 out of 19 ; ages $9,11,13$, and 14) had a paradoxical facilitation in response to the cTBS protocol (Figure 2). Additional analyses were conducted excluding the seven participants who facilitated and the significant positive correlation between age and duration of response remained $[r(10)=0.62, p<0.05]$ with a Mean duration of $49.2 \mathrm{~min}(\mathrm{SD}=33.8 \mathrm{~min})$. The subgroup of participants who displayed the paradoxical facilitation response was not predicted by comorbid symptoms or medication $\left(\chi^{2}=0.091, p=0.76\right)$.

\section{DISCUSSION}

As the application of rTMS protocols to children increases, it is critical to evaluate the safety and tolerability of this procedure in these vulnerable populations. In the current study, all participants tolerated the stimulation and reported only minor discomforts that resolved quickly following the procedures. These findings add to the literature suggesting that rTMS is safe and well tolerated in children and in individuals with ASD (Garvey and Gilbert, 2004; Frye etal., 2008; Croarkin et al., 2011; Wu et al., 2012; Oberman et al., 2013; Rajapakse and Kirton, 2013). Systematic monitoring and documentation of side effects is

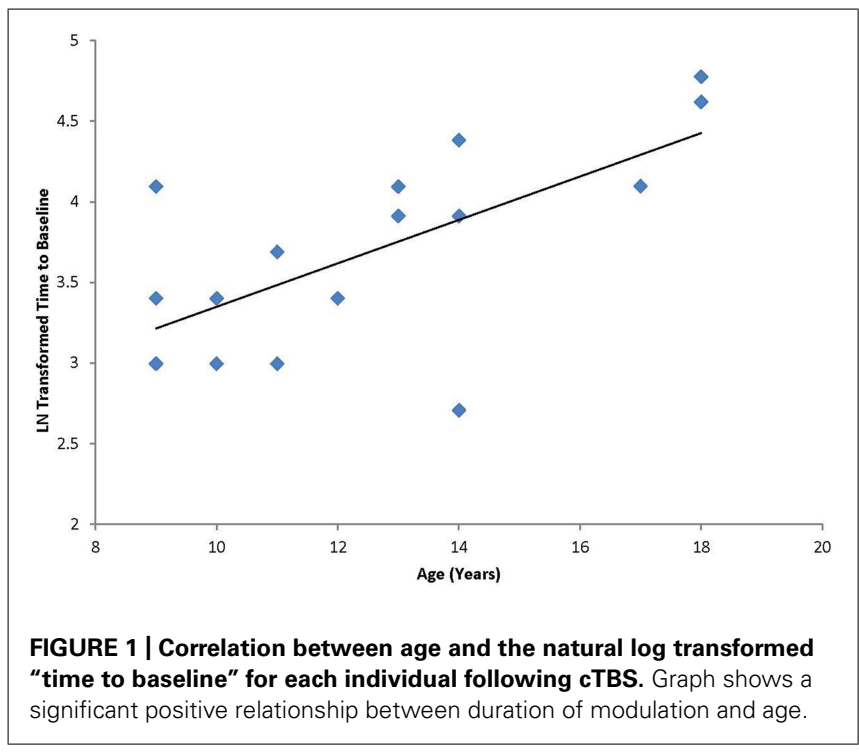




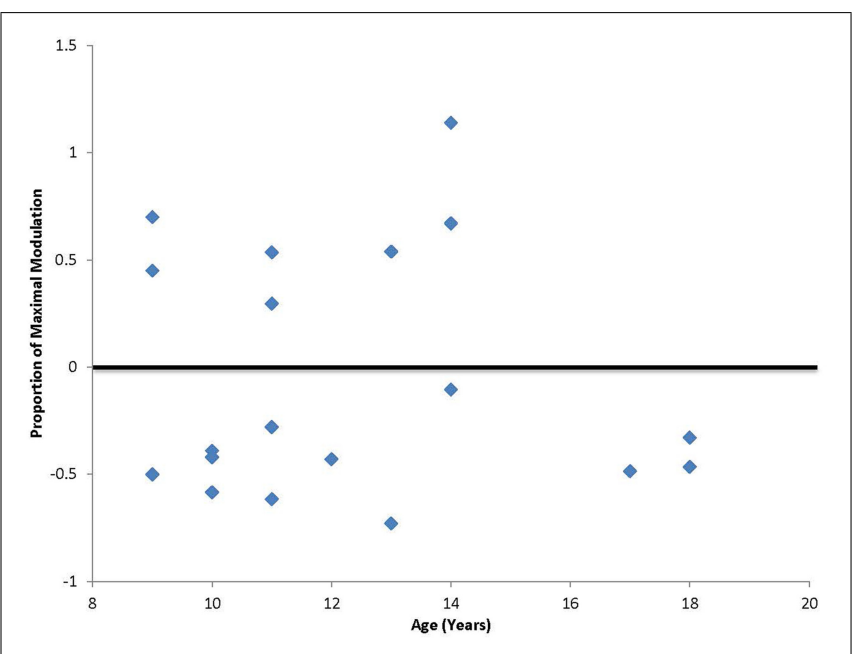

FIGURE 2 | Degree of maximal suppression or facilitation following

cTBS. Values falling above zero indicate paradoxical facilitation in response to the typically suppressive cTBS protocol. Seven out of 20 participants in this study, ages $9,11,13$, and 14 showed this paradoxical facilitation.

critical moving forward to ensure that both participants and investigators have an accurate sense of both the range and frequency of side effects of rTMS in clinical and pediatric populations.

Our findings are also the first step toward the study of the developmental regulation of the cTBS effect and reveal a positive linear relationship between age and duration of modulation of cTBS. Specifically we found that the older participants had a longer lasting response. On the surface this may appear counter intuitive if we consider that response to TBS has been used by our group and others to indicate degree of plasticity. One would imagine that younger children have a greater, not lesser, capacity for plasticity (Huttenlocher, 2002). However, cTBS is thought to model LTD-like plasticity (Huang et al., 2008) and is related to GABAergic inhibitory tone. Thus, perhaps LTD-like plasticity or GABAergic inhibition increases over development, especially during adolescence (Selemon, 2013). As we did not perform iTBS, we cannot speak to the development of LTP-like plasticity in this sample, however, it would be important to evaluate this process as well. Additionally, the current study did not include a sham control condition or any other rTMS protocol, thus it is unclear whether the results are specific to the cTBS paradigm.

Recently, the molecular mechanisms underlying the changes in cortical excitability induced by cTBS have been studied using MRS (Stagg et al., 2009). The findings reveal that the effects of cTBS are mediated by changes in the local activity of inhibitory interneuronal cortical pathways (as measured by changes in cortical GABA concentration in the primary sensorimotor cortex; Stagg etal., 2009). Consistent with the idea that younger children have less inhibitory tone, studies using paired pulse measures of intracortical inhibition have found that children display decreased levels of suppression as compared to adolescents or adults (Walther et al., 2009). This study further claimed that reduced GABA mediated intracortical inhibition may facilitate excitatory (LTP-like) cortical plasticity and motor learning in children. Thus, the current results, although obtained from individuals with ASD, provide further evidence of increasing capacity for LTD-like suppression of cortical excitability across childhood.

Additionally, the finding that over one third of our sample had a paradoxical facilitatory response to cTBS supports the notion of GABAergic dysfunction in ASD. During typical development, GABA currents shift from excitatory to inhibitory through a maturation of chloride transport mechanisms and an age-dependent reduction of intracellular chloride concentration [( $\mathrm{Cl}^{-}{ }_{\mathrm{i}}$; Ben-Ari et al., 2007)]. However, a recent study finds that two ASD animal models (rodent valproate and fragile $\mathrm{X}$ models) show excitatory GABA activity well beyond the age where wild-type animals' GABA activity has shifted to inhibition (Tyzio et al., 2014). In these animals administration of a GABA agonist (isoguvacine) led to an increase in spike frequency in neurons recorded from hippocampal slices as compared to a decrease in wild-type animals. Additionally, the in utero administration of bumetanide, a chloride importer antagonist that reduces intracellular chloride accumulation thereby promoting the shift of GABA from excitation to inhibition, resulted in the restoration of typical electrophysiological and behavioral phenotypes in affected offspring (Tyzio et al., 2014). These preclinical data support the hypothesis that a dysfunction in this shift may contribute to the pathophysiology of ASD (Ben-Ari et al., 2012). Ben-Ari and colleagues have proposed that this dysfunction may be a result of increased intracellular $\left(\left[\mathrm{Cl}^{-}\right]_{\mathrm{i}}\right)$ concentrations in individuals with ASD. This is further supported by a study reporting paradoxical increases in hyperactivity in six out of seven and aggression in seven out of seven children with ASD who were treated with diazepam (Marrosu et al., 1987). Furthermore, in a recent clinical trial where bumetanide, was given to children with ASD results showed improvement in ASD symptoms as measured by the Childhood Autism Rating Scale (CARS) and the Repetitive and Restricted Behavior Scale (RRB) as well as a reduction in aberrant behavior as measured by the Aberrant Behavior Checklist (ABC; Lemonnier and Ben-Ari, 2010). Our findings suggest that one could do an analogous study in humans to explore whether bumetanide would normalize the cTBS modulation in those with a paradoxical facilitation and if this normalization corresponded to improved behavioral symptoms.

We recently suggested that the neurological and behavioral ASD phenotypes are associated with altered brain plasticity that can be measured noninvasively by TMS (Oberman et al., in press). As our data showing age-dependence of the cTBS response suggest, the timing of plastic brain changes may be important for optimal development of cortical circuitry. As ASD is a developmental disorder it would be critical to evaluate the developmental trajectory of abnormalities in a putative mechanism underlying the phenotype.

As the current study did not include healthy control participants or females, it is not clear whether the observed developmental trajectory shown by these males with ASD is similar to what may be obtained in neurotypical individuals or females with ASD. It is possible that variables such as head size or myelination 
could have led to the observed correlation with age. However, as reviewed above, multiple lines of evidence point toward aberrant GABAergic transmission in ASD. Thus, it will be important to evaluate these measures in a healthy developing population and female ASD population to compare the typical developmental trajectory to that shown in males with ASD. Additionally, followup translational studies, analogous to what has been done in animal models, directly testing the relationship between GABA receptor expression [measured by $\left[{ }^{11} \mathrm{C}\right]$ FMZ PET (Maziere et al., 1984)] or concentration [measured by MRS (Mescher et al., 1998)] and measures of cortical reactivity in humans with ASD are needed.

In the current study, we focused on primary motor cortex in the left hemisphere. Thus, it is unclear whether other cortical regions would show similar developmental trajectories or whether there would be a laterality effect in these individuals. The left primary motor cortex was chosen in this study for two reasons. First, MEPs are the standard index used to quantify the effect of TBS protocols. Other indices of cortical excitability outside the motor cortex (e.g., based on electroencephalographic measures) have not yet been well validated for this application. We chose the left hemisphere as it is typically the dominant hemisphere for both rightand left-handed individuals. Second, although motor abnormalities are not considered core symptoms of ASD, many studies have reported motor deficits in individuals with ASD, including alterations in motor milestone development (Teitelbaum et al., 1998), clumsiness, motor incoordination, disturbances in reach-to-grasp movement (Miyahara et al., 1997; Ghaziuddin and Butler, 1998; Mari et al., 2003), deficits in gross and fine motor movement (Noterdaeme et al., 2002), and impaired postural control (KohenRaz et al., 1992; Minshew et al., 2004). It has also been suggested that these motor deficits may underlie the core deficits in ASD (Mostofsky and Ewen, 2011).

Our results support the safety and tolerability of TBS in the pediatric ASD populations. As we continue to enhance our understanding of the relationship between the response to cTBS with GABAergic inhibition and GABAergic dysfunction with ASD pathophysiology we suggest that cTBS may be a practical biomarker of GABAergic dysfunction in this population.

\section{AUTHOR CONTRIBUTIONS}

Lindsay M. Oberman designed the study, collected the data, analyzed the data, and wrote the manuscript. Alvaro Pascual-Leone contributed to the study design, interpretation of the data, and the writing of the manuscript, Alexander Rotenberg contributed to the study design, interpretation of the data, supervised and contributed to data collection, and contributed to writing of the manuscript.

\section{ACKNOWLEDGMENTS}

Work on the project is supported grants the Boston Children's Hospital Translational Research Program (Alexander Rotenberg), National Institutes of Health and National Institute of Mental Health (1R01MH100186), and Harvard Catalyst, The Harvard Clinical and Translational Science Center (NCRR and the NCATS NIH 8KL2TR000168-05). Lindsay M. Oberman is further supported by grants from the Harvard Clinical and
Translational Science Center (8UL1TR000170-05), the Epilepsy Research Foundation, the Simons Foundation and the Nancy Lurie Marks Family Foundation. Alvaro Pascual-Leone is further supported by grants from the National Institutes of Health (R01HD069776, R01NS073601, R21 MH099196, R21 NS082870, R21 NS085491, R21 HD07616, UL1 RR025758), Michael J. Fox Foundation and Sidney R. Baer Foundation. Alexander Rotenberg is further supported by the Center for Integration of Medicine and Innovative Technology (CIMIT), Department of Defense PR121509, Autism Speaks Grant \#8702, and grants from Eisai Inc. and the Epilepsy Research Foundation and epilepsy therapy project.

The content is solely the responsibility of the authors and does not necessarily represent the official views of Harvard Catalyst, Harvard University and its affiliated academic health care centers, or any of the listed granting agencies.

\section{REFERENCES}

APA. (2013). Diagnostic and Statistical Manual of Mental Disorders, 5th Edn. Arlington, VA: American Psychiatric Publishing.

Barker, A. T., Jalinous, R., and Freeston, I. L. (1985). Non-invasive magnetic stimulation of human motor cortex. Lancet 1, 1106-1107. doi: 10.1016/S01406736(85)92413-4

Benali, A., Trippe, J., Weiler, E., Mix, A., Petrasch-Parwez, E., Girzalsky, W., et al. (2011). Theta-burst transcranial magnetic stimulation alters cortical inhibition. J. Neurosci. 31, 1193-1203. doi: 10.1523/JNEUROSCI.1379-10.2011

Ben-Ari, Y., Gaiarsa, J. L., Tyzio, R., and Khazipov, R. (2007). GABA: a pioneer transmitter that excites immature neurons and generates primitive oscillations. Physiol. Rev. 87, 1215-1284. doi: 10.1152/physrev.00017.2006

Ben-Ari, Y., Khalilov, I., Kahle, K. T., and Cherubini, E. (2012). The GABA excitatory/inhibitory shift in brain maturation and neurological disorders. Neuroscientist 18, 467-486. doi: 10.1177/1073858412438697

Blatt, G. J., and S. H. Fatemi (2011). Alterations in GABAergic biomarkers in the autism brain: research findings and clinical implications. Anat. Rec. (Hoboken) 294, 1646-1652. doi: 10.1002/ar.21252

Borghetti, D., Sartucci, F., Petacchi, E., Guzzetta, A., Piras, M. F., Murri, L., etal. (2008). Transcranial magnetic stimulation mapping: a model based on spline interpolation. Brain Res. Bull. 77, 143-148. doi: 10.1016/j.brainresbull.2008.06.001

Cardenas-Morales, L., Gron, G., and Kammer, T. (2011). Exploring the after-effects of theta burst magnetic stimulation on the human motor cortex: a functional imaging study. Hum. Brain Mapp. 32, 1948-1960. doi: 10.1002/hbm.21160

Casanova, M. F., Buxhoeveden, D. P., and Brown, C. (2002). Clinical and macroscopic correlates of minicolumnar pathology in autism. J. Child Neurol. 17, 692-695. doi: 10.1177/088307380201700908

Cherubini, E., Gaiarsa, J. L., and Ben-Ari, Y. (1991). GABA: an excitatory transmitter in early postnatal life. Trends Neurosci. 14, 515-519. doi: 10.1016/0166-2236(91)90003-D

Coghlan, S., Horder, J., Inkster, B., Mendez, M. A., Murphy, D. G., and Nutt, D. J. (2012). GABA system dysfunction in autism and related disorders: from synapse to symptoms. Neurosci. Biobehav. Rev. 36, 2044-2055. doi: 10.1016/j.neubiorev.2012.07.005

Croarkin, P. E., Wall, C. A., and Lee, J. (2011). Applications of transcranial magnetic stimulation (TMS) in child and adolescent psychiatry. Int. Rev. Psychiatry 23, 445-453. doi: 10.3109/09540261.2011.623688

Enticott, P. G., Kennedy, H. A., Rinehart, N. J., Tonge, B. J., Bradshaw, J. L., and Fitzgerald, P. B. (2013). GABAergic activity in autism spectrum disorders: an investigation of cortical inhibition via transcranial magnetic stimulation. Neuropharmacology 68, 202-209. doi: 10.1016/j.neuropharm.2012. 06.017

Fatemi, S. H., Folsom, T. D., Reutiman, T. J., and Thuras, P. D. (2009a). Expression of GABA(B) receptors is altered in brains of subjects with autism. Cerebellum 8 , 64-69. doi: 10.1007/s12311-008-0075-73

Fatemi, S. H., Halt, A. R., Stary, J. M., Kanodia, R., Schulz, S. C., and Realmuto, G. R. (2002). Glutamic acid decarboxylase 65 and $67 \mathrm{kDa}$ proteins are reduced 
in autistic parietal and cerebellar cortices. Biol. Psychiatry 52, 805-810. doi: 10.1016/S0006-3223(02)01430-0

Fatemi, S. H., Reutiman, T. J., Folsom, T. D., Rooney, R. J., Patel, D. H., and Thuras, P. D. (2010). mRNA and protein levels for GABAAalpha4, $\alpha 5, \beta 1$ and GABABR1 receptors are altered in brains from subjects with autism. J. Autism Dev. Disord. 40, 743-750. doi: 10.1007/s10803-009-0924-z

Fatemi, S. H., Reutiman, T. J., Folsom, T. D., and Thuras, P. D. (2009b). GABA(A) receptor downregulation in brains of subjects with autism. J. Autism Dev. Disord. 39, 223-230. doi: 10.1007/s10803-008-0646-647

Freitas, C., Perez, J., Knobel, M., Tormos, J. M., Oberman, L., Eldaief, M., et al. (2011). Changes in cortical plasticity across the lifespan. Front. Aging Neurosci. 3:5. doi: 10.3389/fnagi.2011.00005

Frye, R. E., Rotenberg, A., Ousley, M., and Pascual-Leone, A. (2008). Transcranial magnetic stimulation in child neurology: current and future directions. J. Child Neurol. 23, 79-96. doi: 10.1177/0883073807307972

Gaetz, W., Bloy, L., Wang, D. J., Port, R. G., Blaskey, L., Levy, S. E., et al. (2014). GABA estimation in the brains of children on the autism spectrum: measurement precision and regional cortical variation. Neuroimage 86, 1-9. doi: 10.1016/j.neuroimage.2013.05.068

Garvey, M. A., and Gilbert, D. L. (2004). Transcranial magnetic stimulation in children. Eur. J. Paediatr. Neurol. 8, 7-19. doi: 10.1016/j.ejpn.2003. 11.002

Ghaziuddin, M., and Butler, E. (1998). Clumsiness in autism and Asperger syndrome: a further report. J. Intellect. Disabil. Res. 42, 43-48. doi: 10.1046/j.13652788.1998.00065.x

Hallett, M. (2007). Transcranial magnetic stimulation: a primer. Neuron 55, 187 199. doi: 10.1016/j.neuron.2007.06.026

Huang, Y. Z., Edwards, M. J., Rounis, E., Bhatia, K. P., and Rothwell, J. C. (2005). Theta burst stimulation of the human motor cortex. Neuron 45, 201-206. doi: 10.1016/j.neuron.2004.12.033

Huang, Y. Z., Rothwell, J. C., Edwards, M. J., and Chen, R. S. (2008). Effect of physiological activity on an NMDA-dependent form of cortical plasticity in human. Cereb. Cortex 18, 563-570. doi: 10.1093/cercor/bhm087

Huang, Z. J. (2009). Activity-dependent development of inhibitory synapses and innervation pattern: role of GABA signalling and beyond. J. Physiol. 587, 18811888. doi: 10.1113/jphysiol.2008.168211

Huerta, P. T., and Volpe, B. T. (2009). Transcranial magnetic stimulation, synaptic plasticity and network oscillations. J. Neuroeng. Rehabil. 6:7. doi: 10.1186/17430003-6-7

Huttenlocher, P. R. (2002). Neural Plasticity. Cambridge: Harvard University Press.

Kobayashi, M., and Pascual-Leone, A. (2003). Transcranial magnetic stimulation in neurology. Lancet Neurol. 2, 145-156. doi: 10.1016/S1474-4422(03) 00321-1

Kohen-Raz, R., Volkmar, F. R., and Cohen, D. J. (1992). Postural control in children with autism. J. Autism Dev. Disord. 22, 419-432. doi: 10.1007/ BF01048244

Lemonnier, E., and Ben-Ari, Y. (2010). The diuretic bumetanide decreases autistic behaviour in five infants treated during 3 months with no side effects. Acta Paediatr. 99, 1885-1888. doi: 10.1111/j.1651-2227.2010. 01933.x

Madsen, K. K., Larsson, O. M., and Schousboe, A. (2008). Regulation of excitation by GABA neurotransmission: focus on metabolism and transport. Results Probl. Cell Differ. 44, 201-221. doi: 10.1007/400_2007_036

Mari, M., Castiello, U., Marks, D., Marraffa, C., and Prior, M. (2003). The reach-tograsp movement in children with autism spectrum disorder. Philos. Trans. R Soc. Lond. B Biol. Sci. 358, 393-403. doi: 10.1098/rstb.2002.1205

Markram, K., and Markram, H. (2010). The intense world theory - a unifying theory of the neurobiology of autism. Front. Hum. Neurosci. 4:224. doi: 10.3389/fnhum.2010.00224

Marrosu, F., Marrosu, G., Rachel, M. G., and Biggio, G. (1987). Paradoxical reactions elicited by diazepam in children with classic autism. Funct. Neurol. 2, 355-361.

Maziere, M., Hantraye, P., Prenant, C., Sastre, J., and Comar, D. (1984). Synthesis of ethyl 8-fluoro-5,6-dihydro-5-[11C]methyl-6-oxo-4H-imidazo [1,5-a] [1,4]benzodiazepine-3-carboxylate (RO 15.1788-11C): a specific radioligand for the in vivo study of central benzodiazepine receptors by positron emission tomography. Int. J. Appl. Radiat. Isot. 35, 973-976. doi: 10.1016/0020-708X(84) 90215-1
Mescher, M., Merkle, H., Kirsch, J., Garwood, M., and Gruetter, R. (1998). Simultaneous in vivo spectral editing and water suppression. NMR Biomed. 11, 266-272. doi: 10.1002/(SICI)1099-1492(199810)11:6<266::AID-NBM530>3.0. $\mathrm{CO} ; 2-\mathrm{J}$

Minshew, N. J., Sung, K., Jones, B. L., and Furman, J. M. (2004). Underdevelopment of the postural control system in autism. Neurology 63, 2056-2061. doi: 10.1212/01.WNL.0000145771.98657.62

Miyahara, M., Tsujii, M., Hori, M., Nakanishi, K., Kageyama, H., and Sugiyama, T. (1997). Brief report: motor incoordination in children with Asperger syndrome and learning disabilities. J. Autism Dev. Disord. 27, 595-603. doi: 10.1023/A:1025834211548

Mostofsky, S. H., and Ewen, J. B. (2011). Altered connectivity and action model formation in autism is autism. Neuroscientist 17, 437-448. doi: $10.1177 / 1073858410392381$

Noterdaeme, M., Mildenberger, K., Minow, F., and Amorosa, H. (2002). Evaluation of neuromotor deficits in children with autism and children with a specific speech and language disorder. Eur. Child Adolesc. Psychiatry 11, 219-225. doi: $10.1007 / \mathrm{s} 00787-002-0285-\mathrm{z}$

Oberman, L., Eldaief, M., Fecteau, S., Ifert-Miller, F., Tormos, J. M., and PascualLeone, A. (2012). Abnormal modulation of corticospinal excitability in adults with Asperger's syndrome. Eur. J. Neurosci. 36, 2782-2788. doi: 10.1111/j.14609568.2012.08172.x

Oberman, L. M., Horvath, J. C., and Pascual-Leone, A. (2010). TMS: using the theta-burst protocol to explore mechanism of plasticity in individuals with Fragile X syndrome and autism. J. Vis. Exp. pii: 2272. doi: 10.3791/ 2272

Oberman, L. M., Rotenberg, A., and Pascual-Leone, A. (2013). Use of transcranial magnetic stimulation in autism spectrum disorders. J. Autism Dev. Disord. doi: 10.1007/s10803-013-1960-1962 [Epub ahead of print].

Oberman, L. M., Rotenberg, A., and Pascual-Leone, A. (in press). "Aberrant brain plasticity in autism spectrum disorders," in Plasticity of Cognition in Neurologic Disorders, eds J. Tracy, B. Hampstead, and K. Sathian (New York: Oxford University Press).

Pascual-Leone, A., Freitas, C., Oberman, L., Horvath, J. C., Halko, M., Eldaief, M., et al. (2011). Characterizing brain cortical plasticity and network dynamics across the age-span in health and disease with TMS-EEG and TMS-fMRI. Brain Topogr. 24, 302-315. doi: 10.1007/s10548-011-0196-198

Petroff, O. A. (2002). GABA and glutamate in the human brain. Neuroscientist 8, 562-573. doi: 10.1177/1073858402238515

Rajapakse, T., and Kirton, A. (2013). Non-invasive brain stimulation in children: applications and future directions. Transl. Neurosci. 4, 217-233. doi 10.2478/s13380-013-0116-3

Rossi, S., Hallett, M., Rossini, P. M., Pascual-Leone, A., and Safety of TMS Consensus Group. (2009). Safety, ethical considerations, and application guidelines for the use of transcranial magnetic stimulation in clinical practice and research. Clin. Neurophysiol. 120, 2008-2039. doi: 10.1016/j.clinph.2009. 08.016

Rubenstein, J. L., and Merzenich, M. M. (2003). Model of autism: increased ratio of excitation/inhibition in key neural systems. Genes Brain Behav. 2, 255-267. doi: 10.1034/j.1601-183X.2003.00037.x

Selemon, L. D. (2013). A role for synaptic plasticity in the adolescent development of executive function. Transl. Psychiatry 3:e238. doi: 10.1038/tp. 2013.7

Stagg, C. J., Wylezinska, M., Matthews, P. M., Johansen-Berg, H., Jezzard, P., Rothwell, J. C., et al. (2009). Neurochemical effects of theta burst stimulation as assessed by magnetic resonance spectroscopy. J. Neurophysiol. 101, 2872-2877. doi: 10.1152/jn.91060.2008

Sutor, B., and Luhmann, H. J. (1995). Development of excitatory and inhibitory postsynaptic potentials in the rat neocortex. Perspect. Dev. Neurobiol. 2, 409-419.

Teitelbaum, P., Teitelbaum, O., Nye, J., Fryman, J., and Maurer, R. G. (1998). Movement analysis in infancy may be useful for early diagnosis of autism. Proc. Natl. Acad. Sci. U.S.A. 95, 13982-13987. doi: 10.1073/pnas.95.23. 13982

Theoret, H., Halligan, E., Kobayashi, M., Fregni, F., Tager-Flusberg, H., and Pascual-Leone, A. (2005). Impaired motor facilitation during action observation in individuals with autism spectrum disorder. Curr. Biol. 15, R84-R85. doi: 10.1016/j.cub.2005.01.022 
Thickbroom, G. W. (2007). Transcranial magnetic stimulation and synaptic plasticity: experimental framework and human models. Exp. Brain Res. 180, 583-593. doi: 10.1007/s00221-007-0991-993

Tyzio, R., Nardou, R., Ferrari, D. C., Tsintsadze, T., Shahrokhi, A., Eftekhari, S., et al. (2014). Oxytocin-mediated GABA inhibition during delivery attenuates autism pathogenesis in rodent offspring. Science 343, 675-679. doi: $10.1126 /$ science. 1247190

Walther, M., Berweck, S., Schessl, J., Linder-Lucht, M., Fietzek, U. M., Glocker, F. X., etal. (2009). Maturation of inhibitory and excitatory motor cortex pathways in children. Brain Dev. 31, 562-567. doi: 10.1016/j.braindev.2009. 02.007

Wu, S. W., Shahana, N., Huddleston, D. A., Lewis, A. N., and Gilbert, D. L. (2012). Safety and tolerability of theta-burst transcranial magnetic stimulation in children. Dev. Med. Child Neurol. 54, 636-639. doi: 10.1111/j.14698749.2012.04300.x

Yip, J., Soghomonian, J. J., and Blatt, G. J. (2007). Decreased GAD67 mRNA levels in cerebellar Purkinje cells in autism: pathophysiological implications. Acta Neuropathol. 113, 559-568. doi: 10.1007/s00401-006-0176-173

Ziemann, U. (2004). TMS induced plasticity in human cortex. Rev. Neurosci. 15, 253-266. doi: 10.1515/REVNEURO.2004.15.4.253
Conflict of Interest Statement: Alvaro Pascual-Leone serves on the scientific advisory boards for Nexstim, Neuronix, Starlab Neuroscience, Neuroelectrics, and Neosync; and is listed as an inventor on several issued and pending patents on the real-time integration of transcranial magnetic stimulation (TMS) with electroencephalography (EEG) and magnetic resonance imaging (MRI). Alexander Rotenberg is listed as an inventor on a patent for apparatus and method of use of TMS in epilepsy. He is a co-founder of Neuromotion Inc.

Received: 16 June 2014; accepted: 28 July 2014; published online: 13 August 2014.

Citation: Oberman LM, Pascual-Leone A and Rotenberg A (2014) Modulation of corticospinal excitability by transcranial magnetic stimulation in children and adolescents with autism spectrum disorder. Front. Hum. Neurosci. 8:627. doi: 10.3389/fnhum.2014.00627

This article was submitted to the journal Frontiers in Human Neuroscience.

Copyright (c) 2014 Oberman, Pascual-Leone and Rotenberg. This is an open-access article distributed under the terms of the Creative Commons Attribution License (CC BY). The use, distribution or reproduction in other forums is permitted, provided the original author(s) or licensor are credited and that the original publication in this journal is cited, in accordance with accepted academic practice. No use, distribution or reproduction is permitted which does not comply with these terms. 